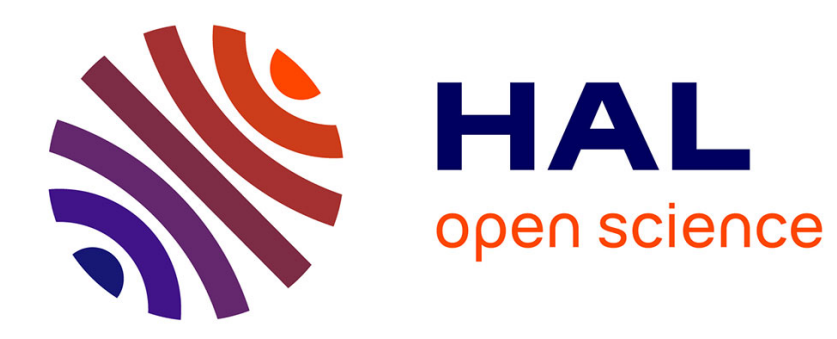

\title{
Inverse problems in the mechanical characterization of elastic arteries
}

Claire Morin, Stéphane Avril

\section{To cite this version:}

Claire Morin, Stéphane Avril. Inverse problems in the mechanical characterization of elastic arteries. MRS Bulletin, 2015, 40 (4), pp.317 - 323. 10.1557/mrs.2015.63 . hal-01380409

\author{
HAL Id: hal-01380409 \\ https://hal.science/hal-01380409
}

Submitted on 13 Oct 2016

HAL is a multi-disciplinary open access archive for the deposit and dissemination of scientific research documents, whether they are published or not. The documents may come from teaching and research institutions in France or abroad, or from public or private research centers.
L'archive ouverte pluridisciplinaire HAL, est destinée au dépôt et à la diffusion de documents scientifiques de niveau recherche, publiés ou non, émanant des établissements d'enseignement et de recherche français ou étrangers, des laboratoires publics ou privés. 


\section{AUTHORS:}

Please follow this template as closely as possible when formatting your article. It contains specific "Styles" in Microsoft Word. If you have any questions, contact the MRS Bulletin editorial office: Lori Wilson, technical editor, lwilson@mrs.org.

\section{Inverse problems in the mechanical characterization of elastic arteries}

Claire Morin*, Stéphane Avril

This article presents an overview of diverse material models used to represent the mechanical behavior of arteries and of the inverse problems posed by the identification of their constitutive parameters. After a brief introduction about the definition of inverse problems and about the general features of arteries, the article addresses three main questions involving inverse problems and arterial wall characterization: (1) macroscopic identification of the parameters of sophisticated constitutive models from traditional uniaxial and biaxial experiments; (2) mesoscopic identification of regional variations in the material parameters of arteries, tracking the effects of functional adaptation or lesions; (3) how constitutive models and inverse problems allow to obtain information on the arterial microstructure and how the structural constituents interact in the mechanical response. Finally, the article shows that a significant effort has been made so far to relate the complex mechanical behavior of arteries to their microstructure but a new class of inverse problems has recently appeared. It is related to the identification of mechanobiological parameters which are the parameters involved in the numerical models of growth and remodeling.

Keywords: inverse problems, constitutive model, arteries, collagen, elastin.

\section{Introduction}

\section{Inverse problems posed by the mechanical characterization of materials}

Identification of mechanical properties is crucial for all kinds of materials since one wants to develop faithful models of solids and structures, predict their mechanical response to a given loading or simply assess their integrity and 
monitor their health. The mathematical problems posed by the identification of material properties are often referred to as inverse problems.

To define an inverse problem, it is convenient to first define its opposite: a forward problem ${ }^{1}$. In mechanics, solving a forward problem means predicting the result of a mechanical action onto a solid (displacement, strain, and stress) from the knowledge of the material model and boundary conditions. One combines the material model and boundary conditions in a boundary-value problem of partial differential equations based on the local mechanical equilibrium. On the other hand an inverse problem is posed when the result of the mechanical action is partly or entirely measured and one wants to employ these measurements to determine unknown parameters of the material model, unknown elements of the boundary conditions or even sometimes the unknown initial geometry of the solid before the mechanical action ${ }^{2}$.

Inverse problems should not be confused with semi-inverse problems. Semi-inverse problems, which are a sub-category of forward problems, have an exact analytical solution, whereas the majority of forward problems have only approximate solutions that can be computed numerically using for instance the finite-element method. Semi-inverse problems occur especially for predicting the result of a mechanical action onto solids having simple geometries ${ }^{3}$. When the result of the mechanical action is measured on such solids and one wants to employ these measurements to determine unknown parameters of the material model, the closed-form expressions of the mechanical fields allow a simpler identification of the unknown material parameters. This subcategory of inverse problems may be named further the semi-forward problems. Semi-forward problems occur in a number of traditional mechanical tests, often called staticallydetermined tests, where the parameters can be estimated by best-fit determination from the data.

Solving inverse problems implies the definition of a cost function, estimating the distance between the model predictions and the measurements. The cost function is minimized in the least-squares sense, by means of a LevenbergMarquardt or a genetic algorithm, except in the case of semi-forward problems 
and linear least-squares, for which an explicit solution can be exhibited. In general situations the model is solved numerically using a finite-element model updating technique (FEMU). In specific situations, when full-field measurements are available, an alternative to FEMU is possible: the Virtual Fields Method, which has been shown to be more robust and efficient in these situations ${ }^{4,5}$.

However, on the contrary to forward problems, a common difficulty of inverse problems is their ill-posed character, which means that existence and uniqueness of the solution are not always guaranteed ${ }^{6}$. The illposedness may be due to a lack of reliable data and/or to an overcomplexity of the model. When access to more reliable data and complexity reduction of the model are not possible in practice, illposedness may be overcome mathematically by resorting to regularization approaches ${ }^{7}$.

\section{Specific context of blood vessels}

The inverse problems, including the semi-forward problems, posed by the identification of material properties in soft biological tissues are not the simplest due to the complex microstructure of soft biological tissues, their large deformation, their response variation by sample and by patient, their anisotropy, their point-dependent non-linear behavior, and their permanent functional adaptation to the environment.

Determining the mechanical properties of such tissues has nevertheless become a field of intense research for the last twenty years since stress analysis in the tissues has been shown to be meaningful for diagnosis in a number of medical applications as for instance in the context of vascular medicine, indicating the risk of rupture of an aneurysm ${ }^{8}$ or the risk of stroke ${ }^{9}$. The present paper is focused especially on the mechanical properties of elastic arteries, which are the largest arteries of the body located closest to the heart (aorta, carotid arteries etc...).

Existing experimental studies for inducing a mechanical stimulus on arterial tissues and for measuring their response are numerous, though out of the scope of this paper. In vitro, many experiments have been developed to characterize pieces of artery after collection on animals or human donors; the most commonplace is the uniaxial tensile test, the biaxial tensile test, the tension- 
inflation test, the bulge inflation test. The deformation may be measured at a single point or as a whole field using an optical technique. In vivo, non-invasive stimuli have to be employed; the most used one being the natural blood action on the arterial wall (pressure variations). A number of techniques have been developed to image the response of arteries to this mechanical action, such as Intravascular Ultrasound Imaging (IVUS) ${ }^{10,11,12}$, Magnetic Resonance Imaging $(\mathrm{MRI})^{13}$, Intravascular Optical Coherence Tomography (OCT) ${ }^{14}$. Some of these techniques are available in the current clinical practice and allow an elastography mode, which actually means that they allow mapping strains at different stages throughout a cardiac cycle.

In all these situations where some elements of the response of an artery subjected to mechanical stimuli are measured, the access to the mechanical parameters is never direct and semi-forward or even inverse problems have to be posed and solved. The rest of the paper is devoted to a survey of these inverse problems in vascular biomechanics, trying to highlight the salient features but also the limits of different identification approaches published so far. After a brief review of the main characteristics of elastic arteries and of their constitutive models, the survey is divided into 3 parts corresponding respectively to 3 major objectives that the researchers try to reach in vascular biomechanics and simultaneously to 3 different length scales of the tissue:

1. Focusing on the macroscopic scale, the first part relates to the general objective of performing (patient-specific) stress analyses on the blood vessels to predict their possible risk of rupture in a context of disease (such as aneurysm, atherosclerotic plaque) or their response to the implantation of a device (stent, graft). Macroscopic constitutive equations are necessary to reach this objective. Arteries are usually modeled by a phenomenological hyperelastic strain energy function involving different numbers of parameters depending on the complexity of the observed behavior. This topic has been a subject of extensive research because of the challenges in identifying parameters that are needed for the sophisticated constitutive equations employed ${ }^{4}, 15,16,17,18,19,20,21,22,23,24,25,26,27,28$, $29,30,31,32$ 
2. Looking further at the mesoscopic scale, the second part relates to the objective of characterizing the regional variations of mechanical properties, often for a comparative qualitative purpose. The inverse problem is posed here by considering heterogeneous distributions of material properties at the scale of the tissue (for instance considering several different layers in the artery).

Characterizing these regional variations is particularly useful for medical diagnosis (since the presence of stiffened regions may indicate a lesion) but also in understanding the progression of diseases and monitoring lesions.

3. Finally at the microscopic scale, the third part relates to the objective of tracking the separate contribution of the different micro-constituents in the global mechanical response as subtle changes in the micromechanical distribution of stresses and strains may alter the basic activity of cells (expression of particular genes, production of particular enzymes and proteins). Due to this permanent activity of growth and remodeling, the tissue is never stress-free and mechanobiology tries to understand the related governing processes.

\section{Inverse problems posed by the complexity of the mechanical behavior at the macroscopic scale}

\section{Generalities about the biomechanics of elastic arteries}

Elastic arteries are soft biological tissues which can be described in terms of their constituents (histological description), of the arrangement of the latter in the microstructure (morphological description) and also as a macroscopic structure subjected to mechanical loading. In terms of histology, elastic arteries are composed of three main types of cells ${ }^{33,34}$ : endothelial cells, smooth muscle cells (SMCs), and fibroblasts, embedded in an extracellular matrix made up mainly of collagen, elastin (in the form of elastic fibers), and a fluid-like ground substance containing among other things proteoglycans. In terms of morphology, arteries are usually arranged in three distinct layers ${ }^{34}$ (see Figure 1). The most inner layer of the vascular wall, called the tunica intima, is delimited from the lumen by the layer of endothelial cells and from the rest of the artery by a fenestrated sheet of elastin called the internal elastic lamina. The intermediate layer, called the tunica media, is made up by SMCs embedded in an extracellular 
matrix made of elastin, collagen and the ground substance. These SMCs are organized in different concentric layers separated by fenestrated elastin sheets. Finally, the outermost layer, called the tunica adventitia, is made of a dense network of collagen fibers, mechanically preventing the wall from overdistension.



Figure 1 : Schematic representation of the arterial wall, showing the three different arterial layers.

Structurally, an elastic artery in vivo is a pre-stretched pipe under an internal pressure load, able to stretch in response to each heart pulse and still able to undergo finite deformations far beyond the ones induced by the pressure variations in the body: while the diameter change between systole and diastole is about $10 \%^{35}$, a segment of artery may shrink from $50 \%$ to $80 \%$ of its length when it is removed from the body ${ }^{36,21,37}$ only due to elastic recoiling.

The different mechanical and organizational features of the arterial wall have been incorporated in diverse phenomenological, macroscopic constitutive models. A comprehensive review of the arterial wall constitutive models can be found in Kalita and Schaefer ${ }^{25}$. Most of the models focus on the passive behavior of arteries (i.e. neglect the mechanical actions of cells) and neglect the viscous effects. We here recall the principal characteristics of the arterial wall models. Because of the large deformability of elastin, the constitutive models for elastic arteries are usually developed within the finite-strain theory and are based on the definition of a strain-energy function. Complex coupling between axial and circumferential responses have always been observed in in vitro experiments. Accounting for that observation, the strain energy function can either be an 
orthotropic exponential strain energy function ${ }^{21}$ or be defined as a fiber-reinforced composite, where each term of the strain energy function accounts for the contribution of a specific constituent ${ }^{23,38,32}$. In these models, elastin and the ground substance are taken into account into the same phase, considered as a neoHookean matrix, and different numbers of collagenous fiber families and SMCs are accounted for by the introduction of polynomial or exponential terms in the strain energy function. The large amount of fluid makes the tissue be almost incompressible ${ }^{16}$. Since the different tissue layers exhibit different mechanical behaviors, a layer-specific strain energy function is sometimes introduced ${ }^{15,29,39}$.

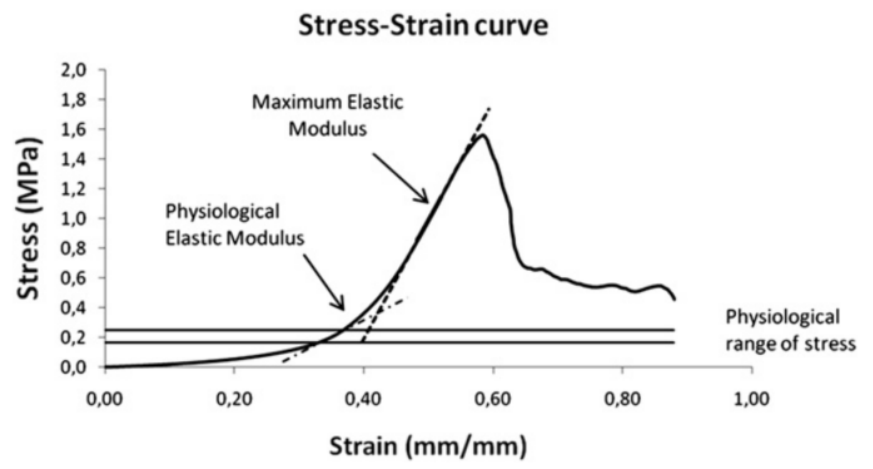

Figure 2 : Uniaxial tensile stress-strain response of a human aortic sample ${ }^{17}$

Due to their permanent functional adaptation, residual stresses sit within the arterial wall. The presence of residual stresses has been evidenced by the observation that the arterial wall opens up in response to a radial cut ${ }^{40,41,42,43,44}$ (see Figure 3). However, empirical observations are not sufficient to measure residual stresses since they are self-equilibrating and complex inverse problems can arise ${ }^{45}$.

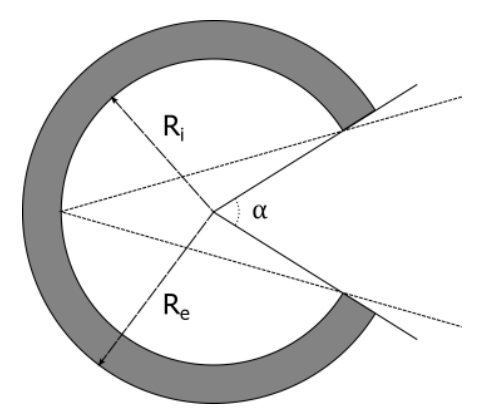

Figure 3: Opening of an arterial wall segment due to a radial cut. 


\section{Inverse problems for characterization of the mechanical properties at the macroscopic scale}

In most cases, statically-determined experimental tests are used to characterize the arteries, leading to semi-forward (inverse) problems. But as already stated in the introduction, due to the complexity of their mechanical behavior, the identification of material parameters of a constitutive model is rarely direct. Different inverse approaches and best-fit methods exist for their identification, depending on the experimental tests that are available for the identification whilst it has been shown that the choice of the cost function can influence the results ${ }^{46}$.

The identification of the parameters of an anisotropic hyperelastic strain energy function requires measuring the response of the material to multiaxial stress loading for different loading paths. The most appropriate tests for arteries are the tension inflation tests which consist in pressure loading the artery at different axial stretches. Auricchio et al. ${ }^{47}$ compared the reliability of two isotropic phenomenological models and of four structural invariant-based constitutive models, commonly used to describe the passive mechanical behavior of arteries, to perform best-fit estimations from the curves of tension inflation tests. The conclusion is that each domain may be reliable depending on the level of local anisotropy in the tissue.

The identification may appear simpler when using uniaxial tests; however, uniaxial tests provide no sensitivity to the mechanical behavior in the other directions of the tissue. In that case, most of the parameters have to be bound in narrow ranges of values in order to overcome the lack of sensitivity. A good example is the study from Masson et al. ${ }^{28}$ who used dynamical intraluminal pressure measured by applanation tonometry to identify the 13 parameters of a material model. Another elegant contribution introducing the notion of state constraints in the minimization problem was performed on a human aorta by Stålhand et al. ${ }^{30}$.

A recent interest concerns the material parameters of the tissue in the regime just preceding its rupture ${ }^{48,49,50,51}$. They usually induce a complex 
identification due to the localized effects of damage preceding rupture but most of the experimental approaches dedicated to this problem so far have assumed homogeneous strains in order to keep the semi-forwardness of the inverse problem.

\section{Inverse problems posed by the regional variations of materials properties at the mesoscopic scale}

As explained in the introduction, elastography is nowadays widely used as a tool for medical diagnosis of different arterial pathologies. Some pathologies such as atherosclerosis are characterized by a local arterial stiffening. In other cases, since the mechanical properties are related to the composition of the tissue, their knowledge helps the doctors to assess the risk of rupture, so as to avoid a stroke or a heart attack. However, elastography strictly allows the mapping of the strain field. Inverse problems have to be solved to determine maps of the mechanical parameters.

In many situations, researchers are only interested in the small deformations of arteries occurring in vivo around a mean static pressure, chosen as the average reference configuration. The small deformations are then induced by the pressure changes in the lumen of the artery between diastole and systole or by palpation. In these situations, the mechanical behavior of the artery is linearized around the reference configuration in such a way that any stress change $\Delta \sigma$ in the artery may induce a strain change such as: $\Delta \varepsilon=C^{-1} \Delta \sigma$. Rigorously, the stiffness tensor $C$ should be an anisotropic tensor, tangent to the stress-strain curve at the reference configuration point, and the equation should only be used for small variations of the strains: $\|\Delta \varepsilon\|<0.05$, for a proper equivalence with the constitutive equations that are characterized from the in vitro bench tests. For larger strains, a polynomial Taylor series expansion is still possible. In a large number of occasions, transverse isotropy is assumed and only the mechanical properties of the artery perpendicularly to the main direction of blood flow are sought. Since the tissue is almost incompressible, a Poisson's ratio is commonly prescribed with values varying from 0.45 to 0.49 and only an elastic modulus $E$ 
has to be identified for a complete material characterization. We would name this modulus the tangent elastic modulus.

Many authors have tried to estimate the regional variations of this parameter, for instance on different sites along the length of an artery or even mapping its distribution across the whole cross section of atherosclerotic plaques in the coronary arteries (using OCT or IVUS) or in the carotid arteries (using MRI). FEMU methods were specifically developed to solve these inverse problems $^{52}$. Nevertheless identifying $E$ is not sufficient to perform a stress analysis on the artery. As the loading applied onto the artery may be dynamic, it may permit to characterize a viscoelastic model ${ }^{18}$.

Note that some authors have also extended the problem of identifying a tangent elastic modulus in a configuration of the artery different from the average in vivo configuration. For instance, some authors have carried out tests in vitro, such as indentation ${ }^{53}$ or micropipette aspiration ${ }^{54}$, on pieces of arteries collected from human donors or animal models. An inverse problem has to be solved to derive the homogeneous or heterogeneous elastic moduli involved in the mechanical response to these tests. However, the obtained values cannot be compared to the in vivo ones as they correspond to linearization of the stressstrain response around two different reference configurations (two different stress states). The main interest of these tangent elastic moduli in configurations different from the in vivo one is to compare different tissues in the same configuration or different locations in the same tissue.

Regional variations of hyperelastic (non-linearized) parameters involved in the complete strain energy functions have rarely been characterized. Variations of material properties across the arterial wall thickness are commonly reported from experiments: independent characterization of the different layers (intima, media, and adventitia) shows their different mechanical properties ${ }^{55}$, which influence the global response of the artery: Humphrey ${ }^{34}$ indeed reported normal and upside-down tension inflation tests on arteries that evidenced their different responses. However, only a few authors ${ }^{15}$ have attempted to solve an inverse problem where both the media and adventitia have unknown material properties 
that have to be extracted from the response of the complete artery. The regional variations of hyperelastic material parameters along the circumferential and axial directions of arteries constitute a new class of inverse problems with a recent interest.

Modeling the arterial wall as a membrane, and taking advantage of the isostaticity of a pressurized membrane, it is possible to reconstruct the (possibly heterogeneous) pointwise stress distribution, without the assumption of a known constitutive behavior ${ }^{56}$. Besides, the strain distribution across the artery can be measured by an optical method. It becomes therefore possible to plot pointwise stress-strain curves and to identify the material parameters point-wisely in a semiforward manner by simple curve fitting. This considerably simplifies the problem, avoiding the repeated resolution of finite element models which is required in other approaches ${ }^{57,58,27}$.

Such an approach has especially been developed for understanding the aneurysm rupture, showing the development of localized damaged zones in the tissue prior to its rupture ${ }^{59}$.

\section{Inverse problems posed by functional adaptation of the arterial tissue at the microscopic scale}

In vascular tissues as in many biological tissues, the physiological properties are closely related to the mechanical environment sensed by the tissues. Indeed, biological tissues have the faculty thanks to mechanosensitive cells to grow and remodel and to adapt their microstructure to new mechanobiological demands. It is also generally agreed upon that the development of vascular pathologies (for instance aneurysms, thrombus...) is highly linked to the remodeling properties of the vascular tissue and to changes in its mechanical environment. Among the different constituents of the vascular wall, collagenous fibers are regularly renewed, while the only period of elastin synthesis by the organism is the perinatal and childhood period, making the elastin degradation be an irreversible process. Besides, the mechanical behavior of vascular tissue is highly complex: reorganization of the microstructure, such as progressive decrimping and reorientation of the elastic and collagenous fibers ${ }^{60,61,62}$ (see 
Figure 4), happens in vascular tissues when subjected to mechanical loading. In order to correctly capture this complex behavior, to account for the remodeling process, and to predict its effect on the overall mechanical behavior, it is necessary to quantify the specific contributions of the mechanically significant constituents to the overall mechanical behavior.


Figure 4 : Progressive decrimping of collagen fibers in the adventitia of a rabbit carotid artery (adapted from Schrauwen et al. ${ }^{62}$. The same area was imaged at different pressure steps from 0 to $140 \mathrm{~mm} \mathrm{Hg}$. The rabbit mean physiological pressure is $70 \mathrm{~mm} \mathbf{H g}$.

The following question can therefore be addressed: what are the predictive capabilities of the available constitutive models to reliably account for separate contributions of the diverse arterial wall constituents on the macroscopic mechanical response ${ }^{63}$ ?

We here restrict ourselves to the passive behavior of arterial wall tissues; we are therefore mainly interested into the two main constituents that contribute to the mechanical response of arterial wall tissues, namely collagen and elastin.

\section{Tracking the contribution of collagen fibers}

The arterial wall owes its main mechanical characteristics, such as the progressive stiffening and anisotropy, to collagen fibers and their orientations ${ }^{39}$. In most of the available constitutive models ${ }^{22,32,64}$, fiber families are 
characterized by their orientation angles while their progressive stiffening is modeled through exponential functions of the stretch. The selection of a constitutive behavior implies the choice of a number of collagen fiber families. The determination of their orientations can be done twofold: either by histological examination of the tissue or by inverse method, searching for the orientation angles that best fit the macroscopic behavior of the tissue. The comparison of both methods shows that the optimal orientation angles stemming from the inverse method are not always consistent with the histological estimations of the fiber orientations both in healthy tissues ${ }^{47}$ and in aneurysmal tissues, for which the inverse method leads to overestimations of the orientation angles ${ }^{65}$. Even if some authors have introduced more complex models including a distribution function of orientations $^{46}$, the great majority of models have a maximum of four fiber families: one circumferentially ${ }^{61}$, one axially, and two diagonally-oriented fiber families. For that matter, histological observations enlighten the difficulty to clearly define families of fiber by allocating them a precise orientation angle ${ }^{47}$.

Modeling the progressive recruitment of collagen fibers is another important question that needs to be addressed. In the (ex-vivo) load-free configuration, microscopic observations evidence crimped fibers with different orientations that the mechanical loading tends to stretch and reorient along the principal strain directions $^{24}$ (see also Figure 4). This progressive reorientation process is generally named the recruitment of collagen fibers. At high stretches, the collagen fibers are perfectly straight and parallel to each other. However, the physiological load lies between these two extreme situations and poses the question of the collagen fiber engagement under physiological conditions. Different experimental studies ${ }^{60,66,67,68}$ showed that only partial engagement of the collagen fibers is reached at physiological pressure: only 5-10\% of the fibers actively participate to the mechanical behavior of vascular tissues at these pressures. This progressive recruitment is the physical origin of the non-linear character and progressive stiffening of the response of vascular tissues; it is generally implicitly accounted for through the introduction of exponential functions in the constitutive models ${ }^{21,39,24,32,21}$, but in some specific models, a 
probability distribution function for the engagement strain of the fibers has been introduced $^{69}$; such a function simplifies the identification of mechanical properties related to the collagen and elastic fibers (thanks to variable recruitment stages)

\section{Tracking the contribution of elastic fibers}

In general, mechanical models only account for the role of elastic fibers (mainly made of elastin) through a neo-Hookean isotropic contribution in the strain energy function. However, biological studies show the importance of elastic fibers in maintaining the shape and the functions of arteries: it is for instance known that arteries with degraded elastin are more prone to local enlargements such as aneurysms ${ }^{70}$. The following question can therefore be posed: which characteristics of the mechanical behavior of elastic fibers can be retrieved from existing models and which ones are missing?

Concerning the mechanical property of elastic fibers, it is generally assimilated to the initial tangent elastic modulus of the arterial stress-strain response. However, evaluation of the neo-Hookean parameter by means of inverse methods and curve fitting algorithm ${ }^{32}$ leads to an elastic modulus for the matrix which is far below the elastic modulus measured on isolated elastic fibers $^{71,20}$. This can be explained by at least two phenomena: first, the matrix in which the collagenous fibers are embedded is not only made of elastic fibers, but also of a ground substance that contributes to the mechanical behavior at low stretches $^{71}$; second, progressive unfolding of the elastic fibers has also been evidenced $^{61}$, impacting the recruitment of collagen fibers: this elastic fiber unfolding occurs before the decrimping of collagenous fibers, which subsequently undergo less decrimping, endowing arterial walls with a more compliant response to pressurization ${ }^{72}$; this is confirmed by observations on arteries of elderly people: their elastin is degraded, and the decrimping of collagenous fibers is more

pronounced and occurs earlier ${ }^{73,74}$. The latter observations clearly evidence strong interactions between the elastic and the collagenous fibers. This strong interaction is not limited to the low-stretch regime, but is also visible at high stretches: models tend to simplify the real behavior of arteries and identify the high-stretch tangent modulus with the one of collagen fibers. However, experiments on 
elastase-treated arteries show a larger elastic modulus for these arteries than for healthy ones, evidencing the contribution of elastic fibers to the mechanical behavior at high stretches ${ }^{20}$.

It must be however stated that inverse determination of the neo-Hookean parameter can be useful for estimation of the quality of elastic fibers, a too low elastic modulus being associated with degraded (non-functional) elastin. In particular, aneurysmal arteries, whose elastin is known to be degraded ${ }^{75}$, exhibit a low initial tangent modulus, as compared to healthy arteries ${ }^{76}$. The importance to consider the contribution of all the components at the microscale and not only the one of the collagen is emphasized by the existence of internal stresses in the tissue that the biomechanicists sometimes try to identify ${ }^{73}$. This constitutes another class of inverse problems for which experimental data are not available yet. For instance, if it would be possible to take the collagen fibers out of the tissue and to measure the deformation that this would induce, this would permit evaluating internal stresses ${ }^{77}$.

\section{Conclusion}

In conclusion, this article showed that the inverse problems posed by the mechanical characterization of arteries are numerous and diverse and it would represent a gigantesque task to make an exhaustive review of all the existing contributions. The more modest purpose of this bulletin was to synthesize the main objectives usually motivating such contributions. Three groups were found: the first one is to have a set of relevant parameters to perform numerical simulations; the second one is to characterize regional variations of material properties to track the effects of functional adaptation or lesions; the third one is to identify the contribution of micro-constituents on the mechanical response.

Even if a relevant theory that can satisfactorily explain the mechanical behavior of soft tissues on the basis of its internal structure and composition is still lacking, a great effort has already been made so far to relate the complex mechanical behavior of arteries to their microstructure and this has motivated numerous inverse problems. The current perspective is however elsewhere, as the 
proper calibration of growth and remodeling models represent nowadays a new challenge. This is a new class of inverse problems related to the mechanobiological characterization of arteries ${ }^{78,79}$ instead of their purely mechanical characterization.

\section{References}

[1] S. Avril, et al., Experimental Mechanics 48, 381 (2008).

[2] F. Riveros, S. Chandra, E. A. Finol, T. C. Gasser, J. F. Rodriguez, Annals of Biomedical Engineering 41, 694 (2013).

[3] J. Criscione, Journal of Elasticity 77, 57 (2004).

[4] S. Avril, P. Badel, A. Duprey, Journal of Biomechanics 43, 2978 (2010).

[5] M. Grediac, F. Pierron, S. Avril, E. Toussaint, Strain 42, 233 (2006).

[6] P. Barbone, N. Gokhale, Inverse Problems 20, 283 (2004).

[7] A. Tikhonov, V. Arsenin, Solutions of Ill-Posed Problems (John Wiley \& Sons, Washington DC, New York, 1977).

[8] M. Fillinger, S. Marra, M. Raghavan, F. Kennedy, Journal of Vascular Surgery 37, 724 (2003).

[9] Z. Li, S. Howarth, T. Tang, J. Gillard, Stroke 37, 1195 (2006).

[10] R. Nishimura, et al., Journal of the American College of Cardiology 16, 145 (1990).

[11] E. Gussenhoven, et al., Journal of the American College of Cardiology 14, 947 (1989).

[12] J. Tobis, et al., Circulation 83, 913 (1991).

[13] D. Woodrum, et al., Magnetic Resonance in Medicine 56, 593 (2006).

[14] H. Yabushita, et al., Circulation 106, 1640 (2002).

[15] P. Badel, S. Avril, S. Lessner, M. Sutton, Computer methods in biomechanics and biomedical engineering 15, 37 (2012).

[16] C. Chuong, Y. Fung, Journal of Biomechanics 17, 35 (1984).

[17] A. Duprey, et al., European Journal of Vascular and Endovascular Surgery 39, 700 (2010). 
[18] H. Eskandari, S. Salcudean, R. Rohling, J. Ohayon, Physics in Medicine and Biology 53, 6569 (2008).

[19] J. Ferruzzi, D. A. Vorp, J. D. Humphrey, Journal of the Royal Society, Interface / the Royal Society 8, 435 (2011).

[20] E. Fonck, et al., American journal of physiology. Heart and circulatory physiology 292, 2754 (2007).

[21] Y. C. Fung, K. Fronek, P. Patitucci, Am J Physiol Heart Circ Physiol 237, H620 (1979).

[22] T. Gasser, R. Ogden, G. Holzapfel, Journal of the Royal Society, Interface / the Royal Society 3, 15 (2006).

[23] G. Holzapfel, H. Weizsäcker, Computers in Biology and Medicine 28, 377 (1998).

[24] G. Holzapfel, Journal of theoretical biology 238, 290 (2006).

[25] P. Kalita, R. Schaefer, Archives of Computational Methods in Engineering 15, 1 (2008).

[26] M. Kauer, V. Vuskovic, J. Dual, G. Szekely, M. Bajka, Medical Image Analysis 6, 275 (2002).

[27] M. Kroon, G. Holzapfel, Computer Methods in Applied Mechanics and Engineering 198, 3622 (2009).

[28] I. Masson, P. Boutouyrie, S. Laurent, J. Humphrey, M. Zidi, Journal of biomechanics 41, 2618 (2008).

[29] A. Pandit, X. Lu, C. Wang, G. S. Kassab, American journal of physiology. Heart and circulatory physiology 288, H2581 (2005).

[30] J. Stalhand, A. Klarbring, M. Karlsson, Biomechanics and Modeling in Mechanobiology 2, 169 (2004).

[31] W. Wan, J. B. Dixon, R. L. G. Jr., Biophysical Journal 102, 2916 (2012).

[32] M. Zulliger, P. Fridez, K. Hayashi, N. Stergiopulos, Journal of Biomechanics 37, 989 (2004).

[33] Y. C. Fung, Biomechanics : Mechanical properties of living tissues (Springer-Verlag, 1993).

[34] J. Humphrey, Cardiovascular Solid Mechanics: Cells, Tissues, and Organs (Springer, New-York, 2002). 
[35] P. Dobrin, Physiological Reviews 58, 397 (1978).

[36] J. Doyle, P. Dobrin, Microvascular Research 3, 400 (1971).

[37] P. Van Loon, W. Klip, E. Bradley, Biorheology 14, 181 (1977).

[38] G. Holzapfel, T. Gasser, M. Stadler, European Journal of Mechanics A/Solids 21, 441 (2002).

[39] G. A. Holzapfel, T. C. Gasser, R. W. Ogden, Journal of elasticity and the physical science of solids 61, 1 (2000).

[40] P. Zeller, T. Skalak, Journal of vascular research 35, 8 (1995).

[41] A. Saini, C. Berry, S. Greenwald, Journal of Vascular Research 32, 398 (1995).

[42] D. Bergel, The visco-elastic properties of the arterial wall., Ph.D. thesis, Queen Mary University of London, UK (1960).

[43] C. J. Chuong, Y. C. Fung, Journal of Biomechanical Engineering 108, 189 (1986).

[44] K. Takamizawa, K. Hayashi, Journal of Biomechanics 20, 7 (1987).

[45] P. Badel, K. Genovese, S. Avril, Strain 48, 528 (2012).

[46] S. Zeinali-Davarani, J. Choi, S. Baek, Journal of biomechanics 42, 524 (2009).

[47] F. Auricchio, M. Conti, A. Ferrara, Archives of Computational Methods in Engineering 21, 273 (2014).

[48] K. Volokh, Journal of Biomechanics 41, 447 (2008).

[49] E. Pena, M. Doblare, Mechanics Research Communications 36, 784 (2009).

[50] T. Gasser, M. Auer, F. Labruto, J. Swedenborg, J. Roy, European Journal of Vascular and Endovascular Surgery 40, 176 (2010).

[51] D. Balzani, J. Schröder, D. Gross, Acta Biomaterialia 2, 609 (2006).

[52] S. Le Floc'h, et al., IEEE Transactions on Medical Imaging 28, 11261137 (2009).

[53] C. Chai, L. Speelman, C. Oomens, F. Baaijens, Journal of Biomechanics 47, 784 (2014). SI: Plaque Mechanics. 
[54] T. Boudou, et al., Journal of Biomechanics 39, 1677 (2006).

[55] G. A. Holzapfel, G. Sommer, C. T. Gasser, P. Regitnig, American Journal of Physiological heart circulation physiology 289, 2048 (2005).

[56] J. Lu, X. Zhou, M. Raghavan, Biomechanics and Modeling in Mechanobiology 7, 477 (2008).

[57] A. Khalil, B. Bouma, M. Kaazempur Mofrad, Cardiovascular engineering (Dordrecht, Netherlands) 6, 93 (2006).

[58] J. Seshaiyer, P.and Humphrey, Journal of biomechanical engineering 125, 363 (2003).

[59] A. Romo, P. Badel, A. Duprey, J. Favre, S. Avril, Journal of Biomechanics 47, 607 (2014).

[60] S. Roy, C. Boss, R. Rezakhaniha, N. Stergiopulos, Journal of biorheology 24, 84 (2010).

[61] H. Wolinsky, S. Glagov, Circulation Research 14, 400 (1964).

[62] J. Schrauwen, et al., Journal of Structural Biology 180, 335 (2012).

[63] J. Humphrey, Journal of mechanics in medicine and biology 9, 243 (2009).

[64] Y. Lanir, Journal of Biomechanics 16, 1 (1983).

[65] J. Kim, S. Avril, A. Duprey, J. Favre, Biomechanics and Modeling in Mechanobiology 11, 841 (2012).

[66] A. Bank, et al., Circulation 94, 3263 (1996).

[67] R. H. Cox, The American journal of physiology 234, 542 (1978).

[68] M. O’Connell, et al., Matrix Biology 27, 171 (2008).

[69] M. Zulliger, N. Stergiopulos, Journal of Biomechanics 40, 3061 (2007).

[70] S. Anidjar, et al., Circulation 82, 973 (1990).

[71] B. B. Aaron, J. M. Gosline, Biopolymers 20, 1247 (1981).

[72] J. Ferruzzi, M. Collins, A. Yeh, J. Humphrey, Cardiovascular research 92, 287 (2011).

[73] S. Zeinali-Davarani, M. Chow, R. Turcotte, Y. Zhang, Annals of biomedical engineering 41, 1528 (2013). 
[74] Z. Samila, S. Carter, Canadian journal of physiology and pharmacology 59, 1050 (1981).

[75] J. Campa, R. Greenhalgh, J. T. Powell, Atherosclerosis 65, 13 (1987).

[76] S. Scott, G. Ferguson, M. Roach, Canadian Journal of Physiology and Pharmacology 50, 328 (1972).

[77] C. Bellini, J. Ferruzzi, S. Roccabianca, E. S. D. Martino, J. D. Humphrey, Annals of Biomedical Engineering 42, 488 (2014).

[78] C. Cyron, J. Humphrey, International Journal of Engineering Science 85, 203 (2014).

[79] J. Wilson, J. Humphrey, Journal of Biomechanics 47, 2995 (2014).

[80] E. Azeloglu, M. Albro, V. Thimmappa, G. Ateshian, K. Costa, American journal of physiology. Heart and circulatory physiology 294, H1197 (2008).

\section{Figure Captions}

Figure 1. Schematic representation of the arterial wall, showing the three different arterial layers.

Figure 2. Uniaxial tensile stress-strain response of a human aortic sample ${ }^{17}$

Figure 3. Opening of an arterial wall segment due to a radial cut.

Figure 4. Progressive decrimping of collagen fibers in the adventitia of a rabbit carotid artery (adapted from Schrauwen et al. ${ }^{62}$. The same area was imaged at different pressure steps from 0 to $140 \mathrm{~mm} \mathrm{Hg}$. The rabbit mean physiological pressure is $70 \mathrm{~mm} \mathrm{Hg}$.

\section{Author biographies}

\section{Claire Morin}

Centre for Biomedical Healthcare and Engineering (CIS-EMSE CNRS:

UMR 5307, LGF), Ecole Nationale Supérieure des Mines, F-42023 Saint-Etienne, France; email claire.morin@mines-stetienne.fr, phone +33477499739.

Claire Morin is an Assistant Professor of biomechanics at Ecole Nationale Supérieure des Mines de Saint-Etienne (France), conducting research in the domain of constitutive behavior and strength of materials. Since 2011, she has specialized in applying the concepts of the homogenization theory to the 
multiscale mechanics of living tissue: first on the elasticity and strength of bones at the Vienna University of Technology; and since 2013 on the mechanical behavior of soft biological tissues.

Stéphane Avril

Centre for Biomedical Healthcare and Engineering (CIS-EMSE CNRS: UMR 5307, LGF), Ecole Nationale Supérieure des Mines, F-42023 Saint-Etienne, France; email stephane.avril @ mines-stetienne.fr, phone +33477420188.

Stéphane Avril is a Full Professor of biomechanics at Ecole Nationale Supérieure des Mines de Saint-Etienne (France) where he is the head of one of the five departments (Center for Biomedical and Healthcare Engineering). Stéphane Avril pioneered inverse methods based on full-field optical methods in solid mechanics between 2003 and 2009 by developing the so-called Virtual Fields method. Since 2010, he has applied these approaches to quantify local distributions of material properties in blood vessels and other soft tissues. After a number of important advances, his research group is now involved in different international projects aimed at promoting patient-specific numerical simulations into the clinical practice. In 2014, Stéphane Avril was a visiting fellow at Yale University (USA). 